\title{
Las relaciones entre los sujetos del sector no estatal cubano: el socio-Trabajador por Cuenta Propia en las Cooperativas No Agropecuarias
} \author{
(Relations between the subjects of the non-state Cuban
} sector: the Self-Employed worker in the Non-Agricultural Cooperatives)

María del Pilar Mesa Mejías ${ }^{1}$

Universidad de Pinar del Río (Cuba)

Sumario: 1. Introducción. 2. El pluriempleo del socio cooperativista como trabajador autónomo. 3. Bibliografía.

Summary: 1. Introduction. 2. The multiple employment of the cooperative member as a self-employed worker. 3. Bibliography.

Resumen: Los gobiernos están obligados a responder a los crecientes problemas sociales que afectan sus sistemas, a través de sus ordenamientos jurídicos, introduciendo figuras como el Pluriempleo y la Pluriactividad, para evitar la paralización del desarrollo del país y garantizar la protección de los derechos de sus ciudadanos. Cuba se encuentra inmersa en un trascendental proceso de actualización del modelo económico, a raíz del 6to Congreso del Partido Comunista, que responde a la necesidad de enfrentar la crisis económica desatada a partir del llamado «Período Especial», consecuencia directa de la influencia de las situaciones que se generaron en el ámbito internacional. Como corolario de dicho proceso, el gobierno cubano determinó fomentar el desarrollo de proyectos negociales particulares o privados, individuales o colectivos, integrados en el sector no estatal de la economía. Aquí encontramos a los Trabajadores por Cuenta Propia y a las Cooperativas No Agropecuarias. Estas dos formas de gestión tienen sus regulaciones propias, donde se establecen las reglas de su funcionamiento. La legislación que regula el sector de las Cooperativas No Agropecuarias, prevé la posibilidad de que se mezclen estas dos, surgiendo relaciones especiales, las que constituyen el centro de investigación del presente trabajo: el socio- Trabajador por Cuenta Propia. Cuba

Palabras clave: cooperativa no agropecuaria; trabajador autónomo;

1 E-mail: marymesa93@gmail.com 
Abstract: Governments are obliged to respond to the growing social problems affecting their systems, through their legal systems, introducing figures such as multiple employment and multiple activity, to avoid the paralysis of the country's development and guarantee the protection of the rights of its citizens. Cuba is immersed in a transcendental process of updating the economic model, following the 6th Congress of the Communist Party, which responds to the need to face the economic crisis unleashed from the so-called "Special Period», a direct consequence of the influence of the situations that were generated in the international arena. As a corollary to this process, the Cuban government decided to encourage the development of private business or projects, individual or collective, integrated into the non-state sector of the economy. Here we find the Self-Employed Workers and the Non-Agricultural Cooperatives. These two forms of management have their own regulations, where the rules of their operation are established. The legislation that regulates the sector of Non-Agricultural Cooperatives, provides for the possibility of mixing these two, emerging special relationships, which constitute the aim of the present paper: the member, Self-Employed Worker. Cuba.

Keywords: Self-Employed Workers; the Non-Agricultural Cooperatives; 


\section{Introducción}

Diversos factores como el crecimiento demográfico, la comercialización a escala planetaria, la industrialización creciente y las crisis económicas, contribuyen en la actualidad al establecimiento de políticas tendentes a conseguir un nivel aceptable de empleo, en aras de mejorar las condiciones de vida de las personas, así como de garantizar el continuo desarrollo del país.

Así, un único trabajo asalariado como fuente de ingresos es más propio de economías asentadas, donde los salarios son altos o llegan con holgura a cubrir las necesidades. Al ser mucho más elevados y precisar un horario laboral regulado, no se necesitan ingresos extras para acumular el mínimo necesario de las familias para subsistir. Luego, en el otro extremo tenemos el desempeño simultáneo de varios empleos, que ha supuesto un añadido esencial en los ingresos de aquellos que viven en condiciones más estrictas de supervivencia, donde la principal aspiración es cubrir las necesidades primarias del hogar, y eventualmente poder aspirar a un ascenso en la escala social.

Esta situación constituye un reto para los sistemas jurídicos de los países, en tanto deben procurar la eficiencia económica de sus instituciones a fin de dar una respuesta válida a los problemas sociales y a los nuevos modelos organizativos de la producción, lo que exige vincular la Economía y el Derecho, poniendo la teoría jurídica en función de las exigencias del sistema económico, aunque sin olvidar los principios de equidad social, ni mucho menos los valores constitucionalmente institucionalizados.

En el Derecho del Trabajo, este fenómeno aparece vinculado al resurgimiento de esquemas negociales individuales dentro de la desregulación y reestructuración flexible del mercado de trabajo. Por esto, la mayoría de los ordenamientos jurídicos prevén figuras favorecedoras de los derechos de los trabajadores, entre estas podemos hacer mención al Pluriempleo y la Pluriactividad, conceptos que se relacionan directamente con el panorama socioeconómico de las naciones.

Cuando hablamos de Pluriempleo y Pluriactividad nos estamos refiriendo a distintas situaciones a las que se hallarán sometidos los trabajadores, tomando como punto de referencia la realización simultánea en el tiempo de varias actividades profesionales por una misma persona.

Ante la situación de crisis por la que atraviesa el mundo en la contemporaneidad, y que afecta directamente a la clase trabajadora, el gobierno cubano comenzó a dar muestras de disposición de evitar lo que en otros países se convierte en un crítico e inminente problema: el desempleo, introduciendo modificaciones en el régimen laboral cubano, 
de lo que constituye un importantísimo ejemplo la entrada en vigor el Decreto Ley 268 «Modificativo del Régimen Laboral», de fecha 29 de junio del 2009, emitido por el Consejo de Estado.

Esta disposición normativa introduce en el Derecho Laboral cubano la figura del Pluriempleo, en aras de estimular las fuerzas productivas, posibilitar la elevación de los ingresos, así como contribuir a que el trabajo constituya la fuente principal de satisfacción de las necesidades materiales y espirituales, con independencia de que el Estado mantenga la protección a quienes lo necesiten.

Paulatinamente se ha generado en el panorama socioeconómico actual un creciente impulso de los proyectos negociales particulares o privados, individuales o colectivos, a los que nosotros preferimos llamar no estatales, en vista de que aquellos son conceptos propios del capitalismo, generado por el proceso de actualización del modelo económico, a raíz del 6to Congreso del Partido Comunista de Cuba, que responde a la necesidad de hacer frente a la crisis económica desencadenada en la década de los años 90, y cuyo resultado principal fue la aprobación de los Lineamientos de la Política Económica y Social del Partido y la Revolución ${ }^{2}$, donde fueron enunciados todos los cambios que se han producido o se efectuarán gradualmente. Uno de ellos consiste en la introducción en la economía de formas de gestión no estatal, y pronto nos hallamos hablando de Trabajadores por Cuenta Propia y Cooperativas No Agropecuarias. En consecuencia, se emitieron las correspondientes regulaciones: para el Trabajo por Cuenta Propia fue la Resolución No. 32 de 7 de octubre de 2010 del Comité Ejecutivo del Consejo de Ministros de Cuba, que fuera derogada y puesta en vigor en la Resolución No. 33 del año 2011. Por otro lado, se implementó la extensión del fenómeno cooperativo a otros sectores de la economía mediante el Decreto Ley $305^{3}$ «De las Cooperativas No Agropecuarias» y el Decreto $309^{4}$ «Reglamento de las Cooperativas No Agropecuarias de primer grado».

2 Lineamientos de la Política Económica y Social del Partido y la Revolución. Aprobados el 18 de abril de 2011. Sexto Congreso del Partido Comunista de Cuba. p. 10: «(...) Los Lineamientos de la Política Económica y Social son la expresión de la voluntad del pueblo, contenida en la política del Partido, el Estado y el Gobierno de la República de Cuba, de actualizar el modelo económico cubano con el objetivo de garantizar la continuidad e irreversibilidad del socialismo, el desarrollo económico del país y la elevación del nivel de vida de la población, conjugado con la necesaria formación de valores éticos y políticos de nuestros ciudadanos(...)».

3 Gaceta Oficial de la República de Cuba No. 053 Extraordinaria, de 11 de diciembre de 2012.

4 Ídem. 
Según estas regulaciones el trabajo por cuenta propia es aquel que no se encuentra subordinado a la administración de una entidad laboral, sino que asume los riesgos de la actividad que autopractica en la forma que estime conveniente y apropiada, con los elementos y materia prima necesarios para su desempeño; mientras que el artículo 2.1 del Decreto-Ley No. $305^{5}$ el legislador nos plantea un concepto de cooperativa en el cual reza:

"La cooperativa es una organización con fines económicos y sociales, que se constituye voluntariamente sobre la base del aporte de bienes y derechos y se sustenta en el trabajo de sus socios, cuyo objetivo general es la producción de bienes y la prestación de servicios mediante la gestión colectiva, para la satisfacción del interés social y el de los socios.»

Sucede que estas formas de gestión no estatal pueden entremezclarse, en tanto un Trabajador por Cuenta Propia puede ser a su vez socio de una Cooperativa No Agropecuaria, y este constituye el eje de nuestra investigación: la generación de una situación de Pluriactividad, no reconocida expresamente por la legislación cubana, lo que dificulta su tratamiento y aplicación adecuados, en tanto existen imprecisiones y contradicciones en la legislación que conllevan a la incorrecta implementación de sus propias disposiciones.

Por esto nuestra investigación va encaminada a determinar cuáles son las imprecisiones e incoherencias, así como las lagunas, que presenta la legislación, las que entorpecen su interpretación y aplicación, resultando útil y necesario su desarrollo para futuras modificaciones de la legislación vigente en materia de cooperativas no agropecuarias, con el objetivo de contar con un régimen legal cooperativo armónico.

\section{El pluriempleo del socio cooperativista como trabajador autónomo}

Según la doctrina ${ }^{6}$ cuando hablamos de Pluriempleo nos estamos refiriendo a la situación del trabajador por cuenta ajena que preste sus servicios profesionales a dos o más empresarios distintos y en actividades que den lugar a su alta obligatoria en un mismo Régimen de la Se-

5 Ídem.

6 Alarte Mayordomo, Carmen María. 2007. «Pluriempleo y Pluriactividad en el sector privado español». Tesis Doctoral. Universidad de Murcia. España. 
guridad Social; mientras que la Pluriactividad hace alusión a la situación del trabajador por cuenta propia y/o ajena cuyas actividades den lugar a su alta obligatoria en dos o más Regímenes distintos del Sistema de la Seguridad Social. Esta última puede ser simultánea, cuando se desarrollan al unísono dos o más actividades profesionales que dan lugar al encuadramiento también simultáneo en dos o más regímenes de la Seguridad Social, o sucesiva cuando estas múltiples actividades no se superponen o solapan en el tiempo.

Las argumentaciones hechas entorno del Pluriempleo han sido mucho más intensas, teniendo en cuenta que la Pluriactividad es una situación más moderna, como explicábamos introductoriamente, generada por las circunstancias actuales a nivel mundial. Para ello ha sido tomado como punto de partida el concepto de la relación jurídico laboral, como aquel vínculo que se establece entre un trabajador y una entidad laboral, según el cual, una parte (el trabajador) se obliga después que se incorpora al colectivo de trabajo correspondiente, a realizar determinado tipo de trabajo, subordinándose al orden laboral interno de la entidad. En tanto que la otra parte (entidad laboral) está obligada a retribuirle su trabajo en correspondencia con su calidad y cantidad, a crear las condiciones de trabajo favorables para su salud y para lograr una alta productividad de trabajo, así como interesarse por sus necesidades materiales y culturales. Podemos concluir que constituye una situación que establece el desempeño de varios cargos, empleos, oficios, etc., por la misma persona, significando una vía de acceso a diferentes entidades mediante la concertación de contratos con cada una, existiendo un contrato principal y uno accesorio.

Partiendo de esta fundamentación podemos establecer entonces que el Pluriempleo es una situación jurídica que solo se refiere a trabajadores por cuenta ajena, que son aquellos cuyas relaciones de trabajo se establecen de manera dependiente con un empleador a cambio de un salario, mientras que la Pluriactividad se aplica tanto a trabajadores por cuenta ajena como a trabajadores por cuenta propia, que en este caso son los que laboran para sí o que pueden tener a su cargo trabajadores contratados, pero son los dueños propios de las ganancias que genera su trabajo. Pueden combinarse una actividad por cuenta propia con una actividad por cuenta ajena, o bien confluir dos actividades por cuenta propia.

Estas situaciones son analizadas fundamentalmente desde la óptica de la contribución a la Seguridad Social. En este sentido, la doctrina española ${ }^{7}$ plantea que es el socio el responsable de elegir el Régimen al

7 ĺdem. 
cual se va a afiliar, ya sea el establecido para la cooperativa, o el de trabajadores autónomos o por cuenta propia, haciéndose constar en los Estatutos esta elección. En caso de elegir la primera opción, corresponde a la cooperativa de forma obligatoria la afiliación, alta, baja y comunicación de variaciones, resultando responsable del ingreso efectivo de la totalidad de las cotizaciones, tanto de su parte como persona jurídica como de la de los socios; mientras que, de elegir la segunda opción, la cooperativa, sin embargo, responderá solidariamente del cumplimiento de la obligación de cotizar que les asiste a los socios, así como de forma subsidiaria por la obligación de afiliación, alta, baja y comunicación de las variaciones de la que responde personalmente el socio.

Lo primordial en este tema es determinar, en principio, si la actividad del socio es una actividad por cuenta propia o ajena. Las proyecciones en torno a este tópico, por un lado esbozan que la actividad que realiza el socio es por cuenta propia en tanto está representando sus propios intereses dado su doble condición de socio-trabajador. Esta relación de mutualidad implica que el socio, como dueño de la cooperativa, deberá apoyar su progreso y vigilar que los otros cumplan con sus obligaciones y responsabilidades, y con las que le asisten a sí mismo dentro de la colectividad; y que como usuario, tiene derecho a todos los servicios y beneficios que la cooperativa implemente, pues la actividad que tiene esta como objeto social estará direccionada justamente hacia ello. Por otro lado, es considerada como una actividad por cuenta ajena teniendo en cuenta que la cooperativa se constituye como una persona jurídica independiente. En relación con este último criterio no existe un consenso en la doctrina respecto a este tema, puesto que depende en gran medida de la naturaleza jurídica que se le reconozca a la institución cooperativa. En este sentido, unos la acogen en sus ordenamientos como sociedad, otros como asociación y otros como una entidad autónoma.

Asimismo, para determinar la ajenidad de la actividad que realiza la persona, se han tenido en cuenta una serie de factores como la "ajenidad de riesgo», fundamentada en que la obligación de asumir los riesgos de la empresa correrá por cuenta del empleador; la relación de dependencia que se establece con respecto a este; y la retribución que recibe el empleado bajo la denominación de salario, definido por el empleador.

Nosotros apoyamos el primer criterio, en tanto el concepto de cooperativa que establece la legislación ${ }^{8}$ deja bien claro que esta se crea

8 vid. p. 4. 
por voluntad propia de sus socios, sobre la base de aportaciones de bienes y derechos, para la satisfacción de sus intereses, así como de la sociedad, pero sobre la base del trabajo de los mismos, resultando este último el argumento más importante para identificar, en primer lugar, la mutualidad, y en segundo lugar, el carácter propio de la actividad que desempeña.

Adentrándonos en el panorama cubano, podemos apreciar la institucionalidad del Pluriempleo, a través del Decreto Ley No.268 «Modificativo del Régimen Laboral», destinado exclusivamente al sector estatal. En este queda estipulado que la situación jurídica del Pluriempleo es aquella en la que «(...) los trabajadores, después de cumplir los deberes del cargo, ocupación o empleo que desempeñan, puedan concertar más de un contrato de trabajo y percibir los salarios que les correspondan por los resultados de la labor realizada.» (Artículo 1 del Decreto Ley No. 268/09 «Modificativo del Régimen Laboral»).

Enmarcando nuestro trabajo investigativo en el sector no estatal, el primer fundamento es puntualizar que las cooperativas no agropecuarias pertenecen junto a los Trabajadores por Cuenta Propia al sector no estatal de la economía, que a nuestra apreciación es la manera socialista de nombrar al sector privado, puesto que este concepto es propio del sistema capitalista: Entraremos entonces a analizar las relaciones internas que se establecen entre los sujetos del mismo sector, o sea, de los Trabajadores por Cuenta Propia con las Cooperativas No Agropecuarias.

Al amparo de la Disposición Especial Primera del Decreto 3099, las Cooperativas No Agropecuarias pueden asociar a Trabajadores por Cuenta Propia. Pretendemos precisar dos momentos en este análisis, uno es el relativo a la afiliación al Régimen de Seguridad Social, como se alude anteriormente, en aras de establecer una comparación con el análisis doctrinal hecho, y un segundo momento para examinar la incidencia de esta relación en la naturaleza y el funcionamiento de la institución Cooperativa.

A partir de esta relación especial, podemos intuir el reconocimiento de la Pluriactividad en nuestro ordenamiento jurídico, que si bien no está instituida de manera expresa, puede apreciarse su presencia al examinar las regulaciones relativas al aporte a la Seguridad Social en las Cooperativas No Agropecuarias. En este sentido, en el artículo 14 del Decreto

9 Gaceta Oficial No. 053 Extraordinaria de 11 de diciembre de 2012. p. 270. " PRIMERA: En las Cooperativas que se constituyan en la modalidad que autoriza el artículo 6, inciso b), del Decreto-Ley, los socios que sean trabajadores por cuenta propia mantendrán debidamente actualizada su respectiva licencia y cumplirán las obligaciones tributarias que como tales les correspondan.» 
Ley 306 «Del Régimen Especial de la Seguridad Social de los socios de las Cooperativas No Agropecuarias», está estipulado de forma precisa que el cooperativista que ejerce simultáneamente una labor comprendida en otro régimen especial de Seguridad Social, puede optar por acogerse a dicho régimen o al que establece el Decreto Ley en cuestión, dirigido a los cooperativistas. En este caso se configura un supuesto de Pluriactividad, si bien no propiamente dicho, pudiera entenderse como tal, pues a pesar de que la legislación obliga a los cooperativistas a afiliarse a su Régimen Especial, exceptúa de este mandato imperativo a los socios- Trabajadores por Cuenta Propia, por cuanto, prevé la posibilidad de que escojan el Régimen al cual se van a acoger, como sucede en la práctica internacional.

Luego, consideramos necesario, esclarecer otras dudas que nos asaltaron al pretender interpretar la legislación. La primera cuestión en la que la normativa es omisa con respecto a si la actividad que está autorizado a realizar ese trabajador por cuenta propia es a fin o no al objeto social de la cooperativa, pues la Disposición Especial Primera del Decreto 309, antes aludida, sólo se refiere a la obligación de mantener debidamente actualizada la licencia que lo ampara a realizar la misma y no incumplir los respectivos deberes tributarios, por lo que al no quedar regulado de manera expresa pudiera entenderse que no necesariamente tiene que ser así, o sea, que puede no corresponderse con el objeto social de la cooperativa de la cual es socio, por cuanto las normas que regulan el Trabajo por Cuenta Propia ${ }^{10}$ permiten obtener más de una licencia, constituyéndose un supuesto de Pluriactividad.

El problema surge en el momento que el socio-Trabajador por Cuenta Propia ejecuta un trabajo para la Cooperativa que sí es afín con el objeto social de esta. ¿Realmente esto constituye una aportación de trabajo, a la que está obligado imperativamente por la legislación, o está rentando su servicio a la Cooperativa? El análisis aquí debe partir del artículo 6 b) del Decreto Ley 30511, el que permite a los asociados

10 Como parte del actual proceso de cambios o «actualización» del modelo económico cubano, consecuentemente con los Lineamientos de la Política Económico y Social del Partido y la Revolución, aprobados en el VI Congreso del PCC en abril de 2011, en octubre de 2010, se publica en la Gaceta Oficial de la República de Cuba en sus números extraordinarios 11 y 12, un conjunto de decretos leyes y resoluciones relacionadas con el trabajo por cuenta propia y el arrendamiento de habitaciones o viviendas particulares.

11 Gaceta Oficial No. 053 Extraordinaria de 11 de diciembre de 2012. p. 250. ARTíCULO 6.- «Las cooperativas de primer grado pueden formarse:

b) Por personas naturales que decidan voluntaria-mente asociarse entre sí, solo con la finalidad de adquirir conjuntamente insumos y servicios, comercializar productos y servicios, o empren-der otras actividades económicas, conservando los socios la propiedad sobre sus bienes.» 
en Cooperativas No Agropecuarias de Primer Grado bajo esta modalidad conservar la propiedad de sus bienes. Luego, si la principal aportación del socio a la Cooperativa lo constituye su trabajo, y esta debe pagarle al socio-Trabajador por Cuenta Propia sólo por el uso de sus bienes destinados a la realización de esa actividad a la cual se dedica, bajo un concepto que tampoco queda definido, pero que en este caso el más razonable sería el arrendamiento, debe ser a través de la licencia por la cual está autorizado a brindar el servicio, entonces, de qué manera se ve aquí la aportación de trabajo a la que está obligado como socio y en la que se sustenta la Cooperativa, si tendrá que ser a través del servicio completo que podrá ofertarle a la cooperativa el uso y disfrute de sus bienes, al ser su titular y por ende conservar el derecho de disposición sobre los mismos.

En este sentido, surgen nebulosas en cuanto a la relación del socioTrabajador por Cuenta Propia con la Cooperativa, ya que existen contradicciones en la legislación al enarbolarse como principio la máxima de que todos los socios están en la obligación de aportar su trabajo, independientemente de cualquier otro aporte que realicen a la Cooperativa No Agropecuaria, en el artículo 4. e) del Decreto Ley 305, que establece el principio de disciplina cooperativista ${ }^{12}$; así como lo dispone expresamente en su artículo 23, al estipular que: «Los socios de la Cooperativa, con independencia de cualquier otro aporte que hagan, están en la obligación de participar con su trabajo.», mientras que en el Decreto 309, se reconoce como un deber de los socios en el artículo 29 b) ${ }^{13}$.

Este planteamiento se ve reforzado por lo establecido en el artículo 65 del Decreto 309 ${ }^{14}$, donde aun cuando se refiere a la contratación de los trabajadores por cuenta propia ajenos a la cooperativa, especifica que el fundamento del contrato está en el servicio que brinda aquel. Así mismo, la Resolución 486/2016 del Ministerio de Finanzas y Precios, la cual modifica el Resuelvo Séptimo de la Resolución No.

12 Gaceta Oficial No. 053 Extraordinaria de 11 de diciembre de 2012. p. 252. Artículo 4. e): « Disciplina cooperativista: Todos los socios aportan su trabajo en la cooperativa; conocen, cumplen y acatan conscientemente las disposiciones que regulan su actividad, así como los acuerdos de sus órganos de dirección y administración, y demás regulaciones que sean de aplicación a la cooperativa.»

13 Gaceta Oficial No. 053 Extraordinaria de 11 de diciembre de 2012. p. 264.

ARTÍCULO 29.- Además de los que se fijen en los estatutos, los socios de la cooperativa tienen los deberes siguientes:

b) aportar su trabajo a la Cooperativa;

14 Gaceta Oficial No. 053 Extraordinaria de 11 de diciembre de 2012. p. 269. ARTíCULO 65.- «El órgano de administración de la Cooperativa pactará los montos a pagar a los trabajadores por cuenta propia por los servicios que se requieran.» 
124 /2016, emitida por el mismo organismo, cuyo Resuelvo Único establece: «Los gastos por la contratación de servicios a los trabajadores por cuenta propia, a las cooperativas no agropecuarias y a otras formas de gestión no estatal se considerarán deducibles hasta el límite del $50 \%$ de los gastos totales en los que incurra la cooperativa.» Aun y cuando estos preceptos se refieren expresamente a los Trabajadores por Cuenta Propia contratados, y no a los que ostentan la condición de socios, es posible interpretar que el Trabajador por Cuenta Propia está relacionado directamente con un servicio externo, dejando abierta la brecha para cuestionarnos si realmente aporta su trabajo a la Cooperativa.

Es evidente la contradicción existente en la propia normativa, en tanto por un lado obliga al socio a contribuir principalmente con su trabajo, y por otro le permite continuar ofertando su servicio como Trabajador por Cuenta Propia, sin distinguir de ninguna manera el sujeto al que le puede brindar el mismo una vez asociado a una Cooperativa No Agropecuaria, lo que nos lleva a interpretar que, efectivamente, pudiera cobrarle a la Cooperativa el servicio, y por ende, no realizar ninguna aportación de trabajo, constituyendo una violación del principio de disciplina cooperativista ${ }^{15}$ enunciado en la misma, como vimos anteriormente.

Esta contradicción se evidencia en la práctica cooperativa actual toda vez que algunas cooperativas, fundamentalmente las de la construcción, que es un sector contratista por naturaleza dadas la amplitud y dinamicidad del mismo, requiere una fuerza de trabajo considerable, que se ve limitada por las disposiciones normativas vigentes, en tanto no pueden contratar más del $10 \%$ del fondo de jornadas- socios ${ }^{16}$, así como no pueden exceder el pago a los Trabajadores por Cuenta Propia en un $50 \%$ de los gastos totales en que incurra la Cooperativa, como ya vimos.

Desde la óptica de la contratación de fuerza de trabajo por la Cooperativa, incluso pudiera pensarse que de alguna manera la legislación puede estar encaminada, en relación con lo anterior, a completar

15 Gaceta Oficial No. 053 Extraordinaria de 11 de diciembre de 2012. p. 250. Artículo 4. e): "Disciplina cooperativista: Todos los socios aportan su trabajo en la cooperativa; conocen, cumplen y acatan conscientemente las disposiciones que regulan su actividad, así como los acuerdos de sus órganos de dirección y administración, y demás regulaciones que sean de aplicación a la cooperativa.»

16 Gaceta Oficial No. 053 Extraordinaria de 11 de diciembre de 2012. p. 252. Artículo 26.2 del Decreto Ley 305: "La contratación a que se refiere el aparta-do anterior no excederá el $10 \%$ del total de las jornadas-socios del período fiscal, conforme establece el Reglamento del presente Decreto-Ley.» 
la fuerza de trabajo necesaria a través de los socios-Trabajadores por Cuenta Propia, por cuanto estos tienen la posibilidad de subcontratar trabajadores, en dependencia de la licencia que lo autoriza a realizar la actividad a la que se dedica, por supuesto; sin embargo, sigue constituyendo una limitación considerable en el sector de la construcción, pues no llega a cubrir las necesidades reales en la ejecución de los trabajos, por la envergadura de los mismos, partiendo de que la licencia de constructor que emite el correspondiente Registro, está en función de determinados objetivos, dígase obras de arquitectura, viviendas hasta cinco niveles, obras industriales y de ingeniería, que por naturaleza requieren un volumen de fuerza de trabajo significativo en la mayoría de los casos, teniendo en cuenta el deterioro de las edificaciones a nivel nacional precisamente por la escasez de recursos y la pésima calidad del trabajo realizado por las entidades constructoras estatales, el mismo que ha propiciado el potenciamiento de las Cooperativas No Agropecuarias en este sector, caracterizadas por la celeridad, calidad y garantía de los servicios que ofrecen.

A manera de conclusión, consideramos que el sistema tributario está bien proyectado, en función de contribuir al presupuesto del Estado, para cubrir aquellos gastos que generan las actividades improductivas que son de su responsabilidad, en tanto se grava a la Cooperativa como persona jurídica a través del Impuesto sobre las Utilidades y por otro lado, al Trabajador por Cuenta Propia, mediante el Impuesto sobre los Ingresos Personales. La problemática entonces, está centrada en el desarrollo de las relaciones que se entablan en el seno de la Cooperativa entre esta y el Trabajador por Cuenta Propia que ostenta a su vez la condición de socio.

Tomando como base el análisis anterior y todos los argumentos expuestos oportunamente en la presente investigación, entendemos necesario ratificar la necesidad imperiosa de modificar el por ciento establecido para la contratación de fuerza de trabajo a que tiene acceso la Cooperativa No Agropecuaria, pues conocemos que este tema ya ha sido objeto de debate en inconmensurables ocasiones, así como hacer una análisis exhaustivo, que pudiera basarse en una comparación con el reconocido Trabajador Autónomo que menciona la doctrina y acogen las legislaciones foráneas, de la relación que se establece entre el socio-Trabajador por Cuenta Propia y la Cooperativa, toda vez que constituye intención expresa del legislador que la misma se sustente en el trabajo de sus socios, en consonancia con el enfoque internacional hacia esta figura, a fin de que se regulen de forma precisa los pormenores relativos a esta relación, propiciando claridad y seguridad jurídicas. 


\section{Bibliografía}

Textos

ALARTE MAYORDOMO, Carmen María. 2007. «Pluriempleo y Pluriactividad en el sector privado español». Tesis Doctoral. Universidad de Murcia. España.

ÁLVAREZ ALCOLEA, Manuel. (s.f). La condición jurídico-laboral de los socios en las cooperativas de producción. (s.l). (s.edit). p. 95- p. 114.

COLECTIVO DE AUTORES, Rivera-Rodríguez, Claudio Alberto. (Comp.). 2009. «Retos y perspectivas del cooperativismo, como real alternativa de desarrollo ante la globalización neoliberal.» Revista Científica Avances, ClGET. Vol. 11 No. 1.

DESDENTADO BONETE, Aurelio y Desdentado Daroca, Elena. (s.f). «En los límites del contrato de trabajo: administradores y socios». Revista del Ministerio de Trabajo e Inmigración 83. (s.l). (s.edit). p. 25- p. 35.

DÍAZ FERNÁNDEZ, Ileana; Pastori, Héctor y Piñeiro Harnecker, Camila. 2012. «El trabajo por cuenta propia en Cuba: lecciones de la experiencia uruguaya.» Boletín cuatrimestral, Economía y gerencia en Cuba: avances de investigación. (Centro de Estudios de la Economía Cubana. Universidad de La Habana). p. 2- p. 7.

ENDARA, Sebastián. «El cooperativismo: Una vía práctica hacia el Buen Vivir». Recuperado en: http://www2.scielo.org.ve/scielo.php?script=sci_ arttext\&pid=S1317-57342008000100001\&lng=es\&nrm=i.

ESPAÑOL MARTÍ, Vicente. «La doble condición de socio y trabajador en las cooperativas de trabajo asociado.» Recuperado en: http://cederul.unizar. es/noticias/sicoderxi/08.htm.

« ¿Es posible trabajar a la vez por cuenta ajena y por cuenta propia?» Recuperado en: http://www.ventanillaempresarial.org/opencms/opencms/es/DudasFrecuentes/Laboral/Laboral.

GUERRA, Pablo. (s.f) «La discusión salarial en el cooperativismo». Uruguay. (s.edit). p. 1- p. 7.

Lineamientos de la Política Económica y Social del Partido y la Revolución. Aprobado el 18 de abril de 2011. Sexto Congreso del Partido Comunista de Cuba.

LÓPEZ I MORA, Frederic. 1999. «Problemática laboral de los socios trabajadores de las empresas de Economía Social: ¿socios o trabajadores?». CIRIECEspaña. Revista de Economía Pública, Social y Cooperativa, n³1, p. 9-46.

MARÍN HITA, Luis. 2014. "Las cooperativas no agropecuarias en Cuba». REVESCO, Revista de Estudios Cooperativos, No 115, p. 120-140.

MOLINA, Nuria. 2015. Se puede ser autónomo y trabajador por cuenta ajena a la vez? Publicado el 18 de agosto de 2015. Recuperado en: http:// infoautonomos.eleconomista.es/blog/ser-autonomo-y-trabajador-porcuenta-ajena/. 
RODRÍGUEZ MUSA, Orestes y Hernández Aguilar, Orisel. 2017. «Apuntes críticos sobre la implementación jurídica del principio cooperativo de «adhesión voluntaria y abierta» en Cuba», Boletín de la Asociación Internacional de Derecho Cooperativo, Núm. 51, pp. 79-98.

TRUJILLO DÍEZ, Iván Jesús. 2002. «Tutela judicial efectiva de los socios trabajadores de cooperativas de producción. A propósito de la STC 86/2002, de 22 de abril». Derecho Privado y Constitución. Núm. 16, p. 393- p. 430.

VUOTTO, Mirta. 2016. Las cooperativas no agropecuarias y la transformación económica en cuba: políticas, procesos y estrategias. REVESCO. Revista de Estudios Cooperativos, núm. 120, p. 149-181.

\section{Legislación}

Gaceta Oficial No. 053 Extraordinaria de 11 de diciembre de 2012: DecretoLey 305 «De las Cooperativas No Agropecuarias»; Decreto-Ley 306 «Del Régimen Especial de Seguridad Social de las Cooperativas No Agropecuarias» y Decreto 309 «Reglamento de las Cooperativas No Agropecuarias de Primer Grado».

Decreto Ley No. 268/09 «Modificativo del Régimen Laboral».

Gaceta Oficial No. 027 Extraordinaria, de 26 de septiembre de 2013. Resoluciones 41 y 42 del Ministerio de Trabajo y Seguridad Social.

Resolución No. 124 /2016 del Ministerio de Finanzas y Precios.

Resolución 486/2016 del Ministerio de Finanzas y Precios. 


\section{Derechos de autor}

El Boletín de la Asociación Internacional de Derecho Cooperativo es una revista de acceso abierto lo que significa que es de libre acceso en su integridad inmediatamente después de la publicación de cada número. Se permite su lectura, la búsqueda, descarga, distribución y reutilización legal en cualquier tipo de soporte sólo para fines no comerciales y según lo previsto por la ley; sin la previa autorización de la Editorial (Universidad de Deusto) o el autor, siempre que la obra original sea debidamente citada (número, año, páginas y DOI si procede) y cualquier cambio en el original esté claramente indicado.

\section{Copyright}

The International Association of Cooperative Law Journal is an Open Access journal which means that it is free for full and immediate access, reading, search, download, distribution, and lawful reuse in any medium only for non-commercial purposes, without prior permission from the Publisher or the author; provided the original work is properly cited and any changes to the original are clearly indicated. 